\title{
Using Data Mining Technique to Predict Cause of Accident and Accident Prone Locations on Highways
}

\author{
Dipo T. Akomolafe ${ }^{1, *}$, Akinbola Olutayo ${ }^{2}$ \\ ${ }^{1}$ Dept. of Mathematical Sciences, Ondo State University of Science and Technology,Okitipupa, Nigeria \\ ${ }^{2}$ Dept. of Computer Science, Joseph Ayo Babalola University,Ikeji Arakeji, Osun State
}

\begin{abstract}
Road accident is a special case of trau ma that constitutes a major cause of disability, untimely death and loss of loved ones as well as family bread winners. Therefore, pred icting the like lihood of road accident on high ways with particular emphasis on Lagos - Ibadan express road, Nigeria in order to prevent accident is very important. Various attempts had been made to identify the cause(s) of accidents on highways using different techniques and system and to reduce accident on the roads but the rate of accident keep on increasing. In this study, the various techniques used to analyse the causes of accidents along this route and the effects of accidents were examined. A technique of using data mining tool to predict the likely occurrence of accident on highways, the likely cause of the accident and accident prone locations was proposed using Lagos -Ibadan highway as a case study. WEKA software was used to analyse accident data gathered along this road. The results showed that causes of accidents, specific time/condition that could trigger accident and accident prone areas could be effectively identified.
\end{abstract}

Keywords Data Mining, Decision Tree, Accident, WEKA, Data Modelling, Id3 Algorithm, Id 3 Tree, Functional Tree Algorithm

\section{Introduction}

Road accident is a special case of trauma that constitutes a major cause of disability and untimely death. It has been estimated that over 300,000 persons die and 10 to 15 million persons are injured every year in road accidents throughout the world. Statistics have also shown that mortality in road accidents is very high among young adults that constitute the major part of the work force. In actual fact, accidents kill faster than AIDS and it gives no preparatory time to its victims. In order to combat this problem, various road safety strategies have been proposed and used. These methods mainly involve conscious planning, design and operations on roads. One important feature of this method is the identification and treatment of accident prone locations commonly called black spots; black spots are not the only cause of accidents on the highway. Also various organizations such as Police High Way Patrol, Vehicle Inspection Officer (VIO), Federal Road Safety Commission (FRSC) among others are charged with the responsibility of maintaining safety thereby reducing road accidents. However, lack of good forecasting techniques has been a major hindrance to these organizations in achieving their objectives.

* Corresponding author:

dtakomolafe@y ahoo.com (Dipo T. Akomolafe)

Published online at http://journal.sapub.org/ database

Copyright (C) 2012 Scientific \& Academic Publishing. All Rights Reserved
It is against this background that Decision Tree is being proposed to model data from road accident database to determine causes of accidents and accident prone locations using historical data collected from Ibadan-Lagos express road as reference point.

\section{Objective}

The primary objective of this research is to use data mining technique; decision tree to predict causes of accident and accident prone locations on highways using data collected on Lagos - Ibadan express way.

\section{Methods}

\subsection{Data Mining}

Data Mining is an interactive process of discovering valid and novel, useful and understandable patterns or models in large database (Han, Mannila and Smyth, 2001). Data Mining, according to Han, Mannila and Symth (2001) is a process that uses a variety of data analys is tools to discover patterns and relationships in data that may be used to make a valid prediction. Data mining uses advances in the field of Artificial Intelligence (AI) and Statistical techniques. Therefore, decision tree is being used in this research

\subsection{Decision Trees}


Decision Trees have emerged as a powerful technique for modelling general input / output relationships. They are tree - shaped structures that represents a series of roles that lead to sets of decisions. They generate rules for the classification of a dataset and a logical model represented as a binary (two - way split) tree that shows how the value of a target variable can be predicted by using the values of a set predictor variables. Decision trees, which are considered in a regression analysis problem, are called regression trees. Thus, the decision tree represents a logic model of regularities of the researched phenomenon.

\subsection{Accidents along Lagos - Ibadan Express Way}

Lagos to Ibadan Express road is one of the busiest roads in Africa. This is because. Lagos was the capital of Nigeria until the seat of government moved to the Federal Capital Territory Abuja and also the headquarters of many national institutions while Ibadan is said to be the largest city in black Africa. The traffic along this route is very heavy because it is a gateway linkage of the heavy traffic going from the Northern, Eastern and Majority of Western states. Fig 3.1 shows the frequency of accidents between the distances of 1 and 40km from Ibadan to Lagos between January 2002 and December 2003. The statistics shows that having a means of predicting likely location of accident base on some input values is essential to advice on dangerous locations.

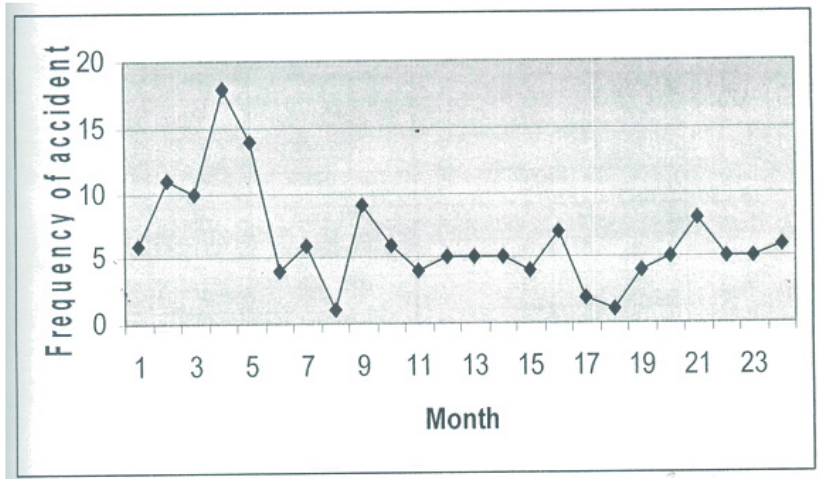

Figure 3.1. Graph of Frequency of Accidents against Month

Several works have been carried out by different researchers both on road accident analysis and forecasting, using Decision Tree and Artificial Neural Networks. Martin, Grandal and Pilkey (2000), analysed the relationship between road infrastructure and safety by using a cross-sectional time-series data base collected for all 50 U.S. states over 14 years. The result suggested that as highway facilities are upgraded, there are reduced fatalities. Gelfand (1991) studied the effect of new pavement on traffic safety in Sweden. The result of his study shows that Traffic accidents increased by $12 \%$ after one year of resurfacing on all types of roads. Akomolafe (2004) employed Artificial Neural Network using multilayer perceptron to predict like lihood of accident happening at particular location between the first 40 kilometers along Lagos-Ibadan Express road and discovered that location 2 recorded the highest number of road accident occurrence and that, tyre burst was the major cause of accident along the route. Oss enbruggen (2005) used a logistic regression model to identify statistically significant factors that predict the probabilities of crashes and injury crashes aiming at using these models to perform a risk assessment of a given region. Their study illustrated that village sites are less hazardous than residential and shopping sites. Abdalla et al (1987) studied the relationship between casualty frequencies and the distance of the accidents from the zones of residence. As might have been anticipated, the casualty frequencies were higher nearer to the zones of residence, possibly due to higher exposure. Ako mola fe et al (2009) used geo spatial technology to identify various positions along major roads in Nigeria. The study revealed that the casualty rates amongst residents from areas classified as relatively deprived were significantly higher than those from relatively affluent areas.

Table 3.1. Record of Accidents along Lagos Ibadan between year 2002 and 2003

\begin{tabular}{|c|c|c|}
\hline S/NO & Month & No of Accident \\
\hline 1 & Jan 2002 & 6 \\
\hline 2 & Feb 2002 & 11 \\
\hline 3 & March 2002 & 10 \\
\hline 4 & April 2002 & 18 \\
\hline 5 & May 2002 & 14 \\
\hline 6 & June 2002 & 4 \\
\hline 7 & July 2002 & 6 \\
\hline 8 & August 2002 & 1 \\
\hline 9 & September 2002 & 9 \\
\hline 10 & October 2002 & 6 \\
\hline 11 & Nov. 2002 & 4 \\
\hline 12 & December 2002 & 5 \\
\hline 13 & Jan 2002 & 5 \\
\hline 14 & Feb 2003 & 5 \\
\hline 15 & March 2003 & 4 \\
\hline 16 & April 2003 & 7 \\
\hline 17 & May 2003 & 2 \\
\hline 18 & June 2003 & 1 \\
\hline 19 & July 2003 & 4 \\
\hline 20 & August 2003 & 5 \\
\hline 21 & September 2003 & 8 \\
\hline 22 & October 2003 & 5 \\
\hline 23 & Nov. 2003 & 5 \\
\hline 24 & December 2003 & 6 \\
\hline
\end{tabular}

\subsection{Process of Data Mining}

The process of data mining consists of three steps which are:

\subsection{Data Preparation}

This includes; Data collection, Data cleaning and Data trans formation.

\subsubsection{Data Modeling}

This research considers the data of accident record between the first $40 \mathrm{~km}$ from Ibadan to Lagos. The data were organized into a relational database.

The unknown causes in Table 3.2 may include other factors such as Law enforcement agent problems, attitude of 
other road users, inadequate traffic road signs, traffic congestion and general vehicle conditions

The sample data used covered the period of 24 Months, that is, January 2002 to December 2003 as indicated in Fig. 3. 1 .

The output variable is the location and the locations can be divided into three distinct reg ions tagged regions $\mathrm{A}, \mathrm{B}$ and $\mathrm{C}$, meaning we have three outputs. Where

First location $1-10 \mathrm{~km}$ is Region A or location 1, Above $10 \mathrm{~km}-20 \mathrm{~km}$ is region $\mathrm{B}$ or Location 2 and above $20 \mathrm{~km}$ is region $\mathrm{C}$ or Location 3
The data sample used covered a period of twenty four Months starting from January 2002 to December 2003. The data were collected by Akomolafe (2004) and this is presented in Table.3.3.

\subsubsection{Deployment}

In this stage, new sets are applied to the model selected in the previous stage to generate predictions or estimates of the expected outcome.

Table 3.2. showing variables given both cont inuous and cat egorical values

\begin{tabular}{|c|c|c|c|c|}
\hline S/N & Variable & Description & Value & Type \\
\hline 1. & Vehicle Type & Small cars & 1 & categorical \\
categorical
\end{tabular}

Table 3.3. Sample Data collected from FRSC (Akomolafe O.P 2004)

\begin{tabular}{|c|c|c|c|c|c|c|c|}
\hline SNO & DATE & TYPE & TIME & SEASON & CAUSE & LOCATION & REG. NO \\
\hline 1 & 6.1 .2002 & 2 & 2 & 1 & 2 & 31 & XG 506 LND \\
\hline 2 & 7.1 .2002 & 2 & 1 & 1 & 1 & 14 & $\mathrm{XC} 720 \mathrm{ACD}$ \\
\hline 3 & 11.1 .2002 & 1 & 1 & 1 & 1 & 14 & AM 713 LND \\
\hline 4 & 12.1 .2002 & 2 & 1 & 1 & 2 & 27 & XE $905 \mathrm{JJJ}$ \\
\hline 5 & 19.1 .2002 & 1 & 2 & 1 & 3 & 27 & AA 559 LAF \\
\hline 6 & 30.01 .02 & 3 & 3 & 1 & 2 & 12 & AA 156 NWD \\
\hline 7 & 03.02 .02 & 2 & 2 & 1 & 2 & 35 & XF 635 JJJ \\
\hline 8 & 05.02 .02 & 2 & 1 & 1 & 2 & 10 & XE 141 AKD \\
\hline 9 & 05.02 .02 & 2 & 3 & 1 & 2 & 14 & XE 124 AKD \\
\hline 10 & 06.02 .02 & 2 & 3 & 1 & 2 & 31 & XE 124 AKD \\
\hline 11 & 11.02 .02 & 1 & 1 & 1 & 3 & 5 & AG 276 LAR \\
\hline 12 & 14.02 .02 & 1 & 1 & 1 & 2 & 14 & \\
\hline 13 & 18.02 .02 & 1 & 2 & 1 & 2 & 18 & \\
\hline 14 & 21.02 .02 & 2 & 1 & 1 & 2 & 19 & XD 249 SMK \\
\hline 15 & 21.02 .02 & 3 & 2 & 1 & 2 & 19 & XC $361 \mathrm{KTU}$ \\
\hline 16 & 24.02 .02 & 2 & 1 & 1 & 2 & 18 & XE 716 SMK \\
\hline 17 & 27.02 .02 & 2 & 3 & 1 & 2 & 35 & XC 307 SGM \\
\hline 18 & 03.03 .02 & 2 & 1 & 1 & 2 & 16 & XE 807 NSR \\
\hline 19 & 05.03 .02 & 1 & 2 & 1 & 2 & 10 & XC 348 AKP \\
\hline 20 & 07.03 .02 & 2 & 1 & 1 & 2 & 2 & OY $2270 \mathrm{JB}$ \\
\hline 21 & 07.03 .02 & 1 & 1 & 1 & 2 & 13 & AP 820 LSD \\
\hline 22 & 07.03 .02 & 3 & 2 & 1 & 2 & 18 & XE 322 APP \\
\hline 23 & 19.03 .02 & 2 & 2 & 1 & 2 & 19 & XC 993 AGL \\
\hline
\end{tabular}




\begin{tabular}{|c|c|c|c|c|c|c|c|}
\hline 24 & 19.03 .02 & & 3 & 1 & 2 & 2 & LA 1804 RF \\
\hline 25 & 30.03 .02 & 1 & 4 & 1 & 2 & 14 & AM 343 FST \\
\hline 26 & 31.03 .02 & 1 & 2 & 1 & 2 & 14 & $\mathrm{KC} 461 \mathrm{ABA}$ \\
\hline 27 & 31.03 .02 & 1 & 2 & 1 & 2 & 14 & BS 142 KJA \\
\hline 28 & 01.04 .02 & 2 & 1 & 2 & 2 & 22 & AA 807 EGB \\
\hline 29 & 01.04 .02 & 1 & 1 & 2 & 2 & 22 & BX 527 GGE \\
\hline 30 & 01.04 .02 & 2 & 2 & 2 & 2 & 18 & AG 787 GNN \\
\hline 31 & 02.04 .02 & 1 & 1 & 2 & 1 & 7 & AU 725 MAP \\
\hline 32 & 02.04 .02 & 2 & 2 & 2 & 2 & 27 & XG 358 APP \\
\hline 33 & 04.04 .02 & 1 & 1 & 2 & 2 & 15 & CY 65 EKY \\
\hline 34 & 04.04 .02 & 1 & 2 & 2 & 2 & 17 & AJ 21 AGG \\
\hline 35 & 05.04 .02 & 1 & 2 & 2 & 1 & 6 & AW 45 FST \\
\hline 36 & 06.04 .02 & 2 & 1 & 2 & 2 & 30 & XB 855 AKD \\
\hline 37 & 07.04 .02 & 1 & 2 & 2 & 1 & 13 & AL 567 YAB \\
\hline 38 & 09.04 .02 & 2 & 2 & 2 & 2 & 12.5 & XA 787 WWP \\
\hline 39 & 13.04 .02 & 2 & 1 & 2 & 1 & 1 & XB $791 \mathrm{GNN}$ \\
\hline 40 & 13.04 .02 & 2 & 1 & 2 & 1 & 11 & XA 127 AFN \\
\hline 41 & 13.04 .02 & 1,2 & 1 & 2 & 1 & 11 & AH $202 \mathrm{AKN}$ \\
\hline 42 & 22.04 .02 & 1 & 2 & 2 & 1 & 15 & RA 01 KRD \\
\hline 43 & 22.04 .02 & 1,3 & 2 & 2 & 1 & 11 & BB $731 \mathrm{KJA}$ \\
\hline 44 & 27.04 .02 & 2 & 2 & 2 & 2 & 27 & AU 739 JJJ \\
\hline 45 & 28.04 .02 & 1 & 2 & 2 & 1 & 14 & AE 316 FST \\
\hline 46 & 03.04 .02 & 1 & 3 & 2 & 2,1 & 12 & AZ 824 AAA \\
\hline 47 & 5.8 .2002 & 1 & 1 & 2 & 2 & 20 & AA 654 GBY \\
\hline 48 & 5.8 .2002 & 1 & 1 & 2 & 2 & 30 & XF 65 JJJ \\
\hline 49 & 5.10 .2002 & $2 \& 1$ & 1 & 2 & 1 & 35 & DM 207 AAA \\
\hline & & & & 2 & & & BL 86 AAA \\
\hline 50 & 5.10 .2002 & 1 & 1 & 2 & $1 \& 2$ & 35 & BR 608 LSR \\
\hline 51 & 5.11 .2002 & 3 & 1 & 2 & 2 & 26 & XB 606 APP \\
\hline 52 & 5.13 .2002 & 2 & 1 & 2 & 1 & 2 & XA 616 YLW \\
\hline 53 & 5.13 .2002 & 1 & 1 & 2 & 1 & 26.5 & BM 566 GGE \\
\hline 54 & 5.14 .2002 & 2 & 3 & 2 & 2 & 15 & XC 348 AKD \\
\hline 55 & 5.15 .2002 & 1 & 2 & 2 & 2 & 19 & OY $2077 \mathrm{JB}$ \\
\hline 56 & 5.15 .2002 & 1 & 2 & 2 & 2 & 14 & AJ $101 \mathrm{NND}$ \\
\hline 57 & 5.20 .2002 & 1 & & 2 & 2 & 26 & AU 682 ABC \\
\hline 58 & 5.21 .2002 & 2 & & 2 & 2 & 24 & XG 719 FST \\
\hline 59 & 5.25 .2002 & 1 & 1 & 2 & 2 & 12 & AV 70 LSR \\
\hline 60 & 6.2 .2002 & & 3 & 2 & 1 & 12 & AZ 191 MUS \\
\hline 61 & 6.3 .2002 & 2 & 2 & 2 & 2 & 16 & AQ $742 \mathrm{YYY}$ \\
\hline 62 & 6.15 .2002 & 2 & 1 & 2 & 2 & 12 & XA 682 YRE \\
\hline 63 & 6.16 .2002 & 1 & 1 & 2 & 2 & 21 & AL $885 \mathrm{AKN}$ \\
\hline 64 & 6.16 .2002 & 2 & 1 & 2 & 2 & 21 & XE 751 SMK \\
\hline 65 & 7.15 .2002 & 2 & 1 & 2 & 3 & 12 & XH 649 GGE \\
\hline 66 & 7.20 .2002 & 2 & 2 & 2 & 2 & 10 & XB 286 KNR \\
\hline 67 & 8.8 .2002 & 3 & 2 & 2 & 2 & 12 & XE 232 SGM \\
\hline 68 & 9.19 .2002 & 1 & 3 & 2 & 2 & 22 & XA $940 \mathrm{KNH}$ \\
\hline 69 & 9.20 .2002 & 2 & 1 & 2 & 2 & 4 & AX 94 JJJ \\
\hline 70 & 9.20 .2002 & 3 & 2 & 2 & 2 & 7 & XC 768 BDJ \\
\hline 71 & 9.21 .2002 & 1 & 1 & 2 & 1 & 29 & BL 254 SMK \\
\hline 72 & 9.21 .2002 & 2 & 1 & 2 & 1 & 16 & AP 647 AKR \\
\hline 73 & 9.21 .2002 & 2 & & 2 & 2 & 18 & XC 253 GGE \\
\hline 74 & 9.22 .2002 & 2 & 1 & 2 & 2 & 10 & LA 979 BG \\
\hline 75 & 9.22 .2002 & 2 & 3 & 2 & 2 & 16 & XU 510 GGE \\
\hline 76 & 9.27 .2002 & & 2 & 2 & 2 & 12 & \\
\hline 77 & 10.1 .2002 & 1 & 2 & 2 & 1 & 6 & AA 05 MHA \\
\hline 78 & 10.14 .2002 & 2 & 1 & 2 & 2 & 13 & XE 869 MUS \\
\hline 79 & 10.16 .2002 & 2 & 2 & 2 & 2 & 15 & XB 888 AKR \\
\hline 80 & 10.29 .2002 & & 2 & 2 & 2 & 7 & \\
\hline 81 & 10.29 .2002 & 2 & 2 & 2 & 2 & 17 & XD 168 BDJ \\
\hline 82 & 10.29 .2002 & 3 & 1 & 2 & 2 & 6 & AA 342 LES \\
\hline 83 & 11.4 .2002 & 2 & 1 & 1 & 1 & 5 & BX 877 KJA \\
\hline 84 & 11.10 .2002 & 2 & 1 & 1 & 2 & 12 & XC 637 RKJ \\
\hline 85 & 11.10 .2002 & 2 & 2 & 1 & 2 & 11 & XC 937 SGM \\
\hline 86 & 11.12 .2002 & 1 & & 1 & & 12 & AA $466 \mathrm{KNR}$ \\
\hline
\end{tabular}




\begin{tabular}{|c|c|c|c|c|c|c|c|}
\hline 87 & 2.12 .2004 & 2 & 1 & 1 & 2 & 14 & XG $182 \mathrm{JJJ}$ \\
\hline 88 & 12.7 .2002 & 3 & 2 & 1 & 2 & 1 & XA 425 CRC \\
\hline 89 & 12.10 .2002 & 2 & 3 & 1 & 3 & 13 & XD 695 EKY \\
\hline 90 & 12.11 .2002 & 2 & 2 & 1 & 2 & 16 & XA 350 EDY \\
\hline 91 & 12.12 .2002 & & 1 & 1 & 2 & 14 & XG $955 \mathrm{KSF}$ \\
\hline 92 & 23.01 .2002 & 1 & 3 & 1 & 1 & 16 & XA 411 EJG \\
\hline 93 & 18.01 .03 & 1 & 3 & 1 & 1 & 18 & AE 015 GBN \\
\hline 94 & 27.01 .03 & 2 & 2 & 1 & 2 & 8 & XD 125 LSR \\
\hline 95 & 29.01 .03 & 3 & 4 & 1 & 2 & 12 & XC 616 KTU \\
\hline 96 & 29.01 .03 & 2 & & 1 & 2 & 14 & XF 797 AKD \\
\hline 97 & 02.02 .03 & 2 & 1 & 1 & 2 & 18 & CW 293 AAA \\
\hline 98 & 12.02 .03 & 1 & 2 & 1 & 1 & 18 & AV 3 GGE \\
\hline 99 & 12.02 .03 & 2 & 2 & 1 & 2 & 18 & XB 6 WWD \\
\hline 100 & 12.02 .03 & 1 & 3 & 1 & 1 & 12 & HB $40 \mathrm{KJA}$ \\
\hline 101 & 17.02 .03 & 2 & 3 & 1 & 2 & 11 & XB $446 \mathrm{MNY}$ \\
\hline 102 & 05.03 .03 & 1 & 2 & 1 & 2 & 6 & AE $753 \mathrm{KRE}$ \\
\hline 103 & 19.03 .03 & 2 & 1 & 1 & 2 & 12 & XH 382 ABC \\
\hline 104 & 28.03 .03 & 3 & & 1 & 1 & 12 & AG 145 NRK \\
\hline 105 & 31.03 .03 & 2 & 3 & 1 & 2 & 13 & AA 499 GBY \\
\hline 106 & 05.04 .03 & 2 & 2 & 2 & 3 & 11.5 & XD 432 KSF \\
\hline 107 & 06.04 .031 & 1 & 1 & 2 & 3 & 12 & CE 188 JJJ \\
\hline 108 & 06.04 .03 & 2 & 1 & 2 & 2 & 12 & FA $01 \mathrm{JJ}$ \\
\hline 109 & 14.04 .03 & 1 & 1 & 2 & & 28 & FV 43 AAA \\
\hline 110 & 24.04 .03 & 1 & 2 & 2 & 2 & 7 & OY $01 \mathrm{SE}$ \\
\hline 111 & 24.04 .03 & 3 & 2 & 2 & 2 & 9 & XB $328 \mathrm{MAG}$ \\
\hline 112 & 30.04 .03 & 3 & 3 & 2 & 1 & 16 & XD 644 NRK \\
\hline 113 & 10.05 .03 & & 1 & 2 & & 40 & AA $399 \mathrm{KTU}$ \\
\hline 114 & 16.05 .03 & 1 & 3 & 2 & 2 & 20 & XH 327 ADC \\
\hline 115 & 02.06 .03 & 1 & 1 & 2 & 1 & 8 & XB 144 YRE \\
\hline 116 & 20.07 .03 & 2 & 1 & 2 & 2 & 27 & 5K $324 \mathrm{LND}$ \\
\hline 117 & 26.07 .03 & 1 & 2 & 2 & 2 & 9 & DG 329 LSR \\
\hline 118 & 28.07 .03 & 2 & 2 & 2 & 2 & 13 & XJ 179 LND \\
\hline 119 & 28.07 .03 & 2 & 2 & 2 & 1 & 18 & XF 114 EPE \\
\hline 120 & 02.08 .03 & 1 & 1 & 2 & 2 & 13 & CB 434 MUS \\
\hline 121 & 02.08 .03 & 1 & 1 & 2 & 1 & 8 & XG 954 FST \\
\hline 122 & 09.08 .03 & 1 & 1 & 2 & 1 & 19 & AG 802 SGB \\
\hline 123 & 16.08 .03 & 2 & 2 & 2 & 2 & 2 & XF $450 \mathrm{SMK}$ \\
\hline 124 & 31.08 .03 & 1 & 1 & 2 & 1 & 14 & OY $1281 \mathrm{TD}$ \\
\hline 125 & 01.09 .03 & 3 & 2 & 2 & 1 & 8 & XA $362 \mathrm{KJA}$ \\
\hline 126 & 08.09 .03 & 1 & & 2 & & 18 & XH $723 \mathrm{JJJ}$ \\
\hline 127 & 14.09 .03 & & & 2 & & 19 & \\
\hline 128 & 16.09 .03 & 1 & 2 & 2 & 2 & 6 & AA 112 YRE \\
\hline 129 & 21.09 .03 & 2 & 1 & 2 & 2 & 31 & XB 766 AGG \\
\hline 130 & 24.09 .03 & 2 & 2 & 2 & 1 & 18 & $\mathrm{XC} 115 \mathrm{EDE}$ \\
\hline 131 & 28.09 .03 & 2 & 1 & 2 & 2 & 14 & XN 739 AAA \\
\hline 132 & 28.09 .03 & 2 & 3 & 2 & 2 & 13 & XD 642 NRK \\
\hline 133 & 06.10 .03 & 1 & 2 & 2 & 2 & 11 & DG 548 LND \\
\hline 134 & 14.10 .03 & 2 & 2 & 2 & 2 & 12 & XA 730 FUF \\
\hline 135 & 18.10 .03 & 2 & 3 & 2 & 2 & 28 & XA $286 \mathrm{GBH}$ \\
\hline 136 & 19.10 .03 & & 1 & 2 & 2 & 22 & AA 188 AAA \\
\hline 137 & 20.10 .03 & 2 & 2 & 2 & 2 & 27 & LG 016 KNE \\
\hline 138 & 01.11 .03 & 3 & 1 & 1 & 1 & 9 & XA $847 \mathrm{KEH}$ \\
\hline 139 & 02.11 .03 & 2 & 2 & 1 & 2 & 18 & XC 575 GGE \\
\hline 140 & 25.11 .03 & 1 & 1 & 1 & 3 & 24 & BO 984 APP \\
\hline 141 & 27.11 .03 & 1 & 1 & 1 & 2 & 18 & AJ 06 SGB \\
\hline 142 & 27.11 .03 & 2 & 2 & 1 & 2 & 13 & XB 369 EKY \\
\hline 143 & 06.12 .03 & 2 & 1 & 1 & 2 & 13 & AP $938 \mathrm{KJA}$ \\
\hline 144 & 09.12 .03 & 3 & 3 & 1 & 1 & 13 & BM $130 \mathrm{MAP}$ \\
\hline 145 & 13.12 .03 & 2 & 1 & 1 & 1 & 7 & XA 610 ARP \\
\hline 146 & 22.12 .03 & 1 & 1 & 1 & 1 & 11 & BL 500 GGE \\
\hline 147 & 24.12 .03 & & 1 & 1 & 3 & 12 & JB 356 KJA \\
\hline 148 & 24.12 .03 & 2 & 2 & 1 & 2 & 13 & XG $562 \mathrm{AKD}$ \\
\hline
\end{tabular}




\section{Results}

\subsection{Analysis}

The major step required to obtain result of the research was carried out by analysing the data using WEKA. WEKA is a collection of machine learning algorithms and data processing tools. It contains various tools for data pre-processing, classification, regression, clustering, association rules and visualization. There are many learning algorithms implemented in WEKA including Bayesian classifier, Trees, Rules, Functions, Lazy classifiers and miscellaneous classifiers. The algorithms can be applied directly to a data set. WEKA is also data mining software developed in JA VA it has a GUI chooser fro $m$ which any one of the four major WEKA applications can be selected. For the purpose of this study, the Explorer application was used.

The Explorer window of W EKA has six tabs. The first tab is pre- process that enables the formatted data to be loaded into WEKA environment. Once the data has been loaded, the preprocess panel shows a variety of information as shown in figure 4.3 below.

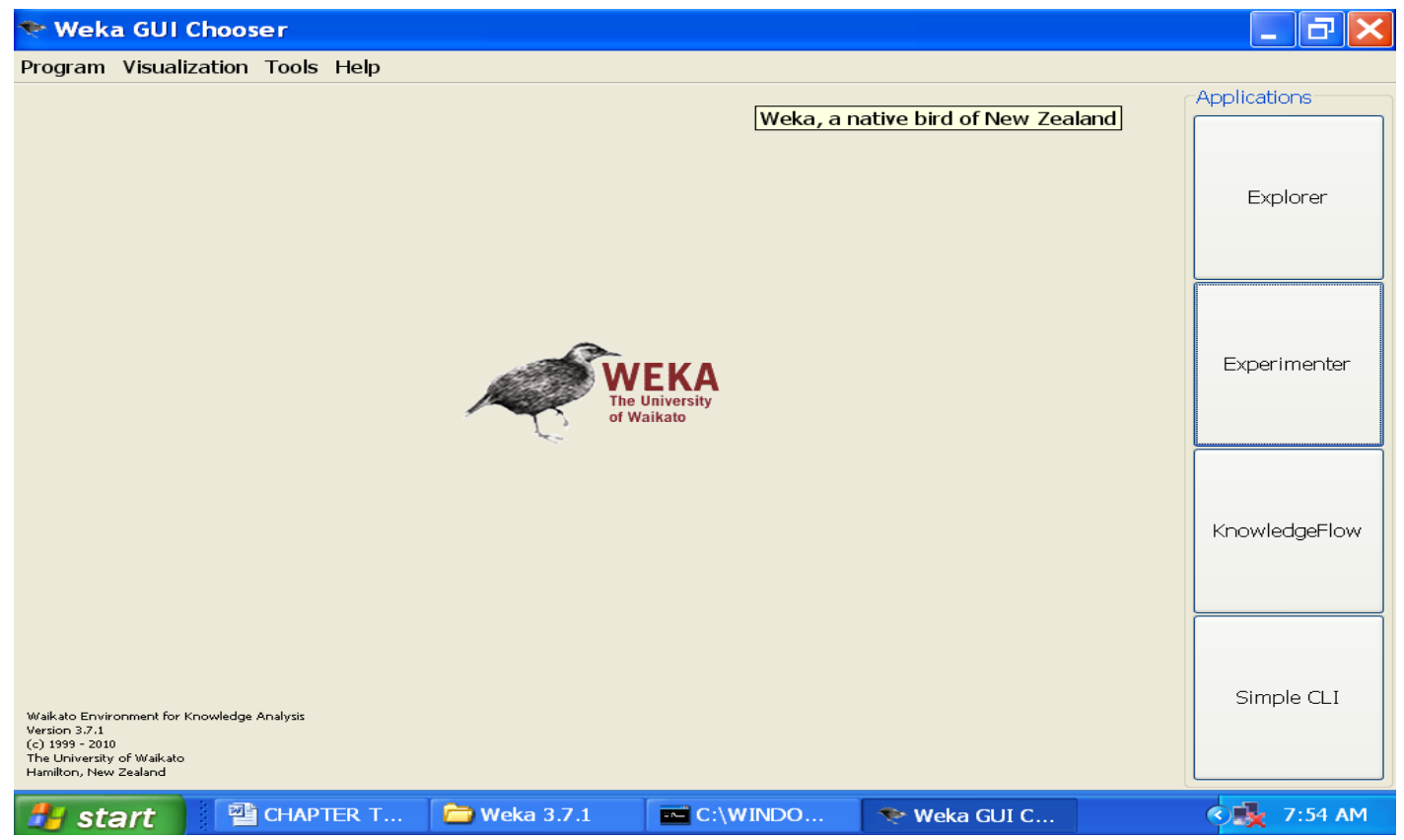

Figure 4.1. WEKA GUI chooser

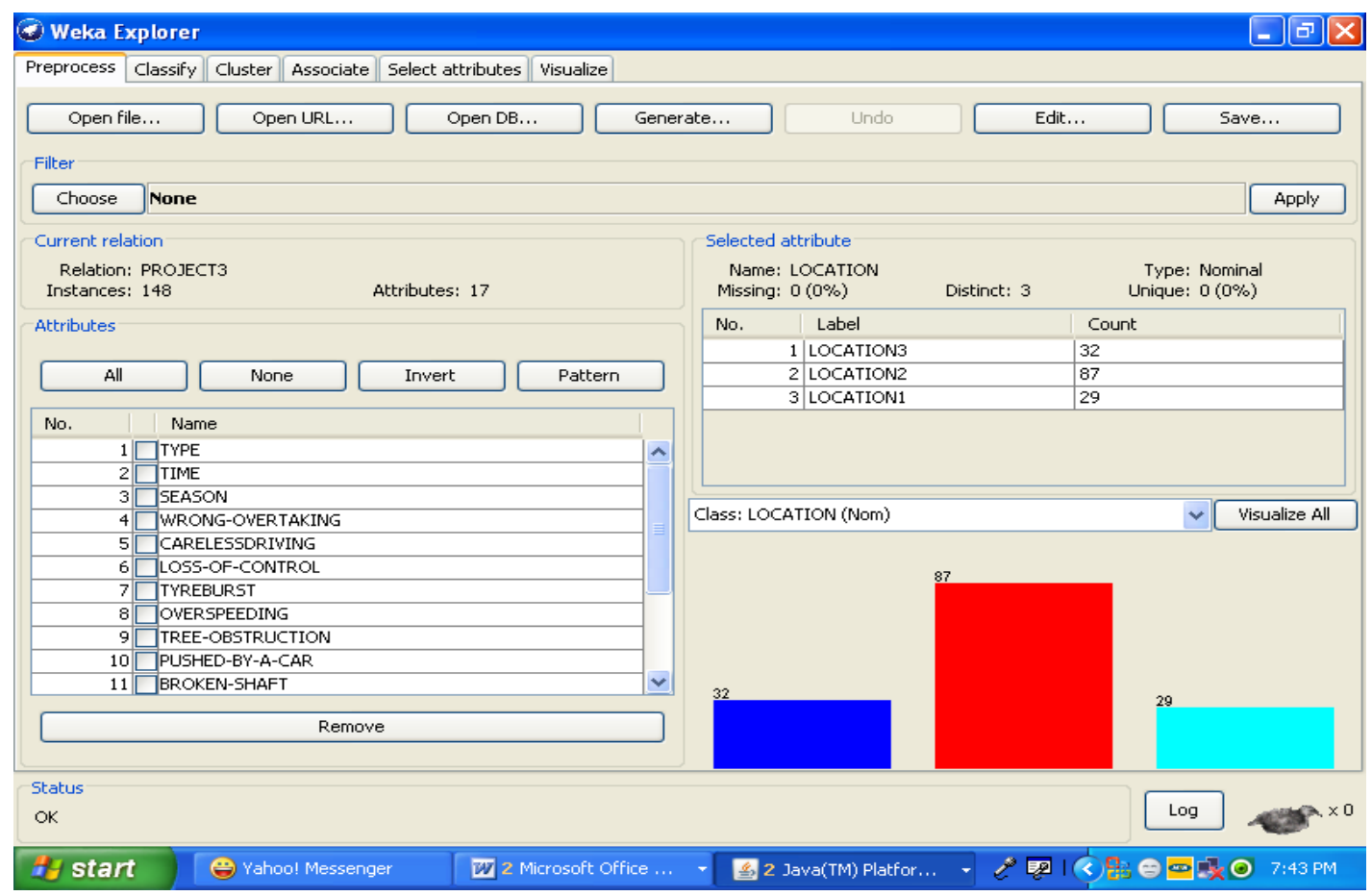

Figure 4.2. WEKA Explorer 


\subsection{Weka Classifiers}

There are several classifiers available in WEKA but Function Tree and Id3 were used in this study in case of Decision Tree. Pris $m$ Rule based learner was generated using WEKA. Attribute importance analysis was carried out to rank the attribute by significance using information gain. Finally, correlation based feature subset selection (cfs) and consistency subset selection (COE) filter algorithm were used to rank and select the attribute that are most useful. The F- measure and the AUC which are well known measures of probability tree learning was used as evaluation metrics for model generated by WEKA clas sifiers.

Several numbers of setups of decision tree algorithms have been experimented and the best result obtained is reported as the data set. Each class was trained with entropy of fit measure, the prior class probabilities parameter was set to equal, the stopping option for pruning was misclassification error, the minimum $\mathrm{n}$ per node was set to 5 , the fraction of objects was 0.05 , the maximum number of nodes was 100 , surrogates was 5, 10 fold cross-validation was used, and generated comprehensive results.

The best decision tree result was obtained with Id3 with 115 correctly classified instances and 33 incorrectly classified instances which represents $77.70 \%$ and $22.29 \%$ respectively.

Mean absolute error was 0.1835 and Root mean squared error was 0.3029 .

The tree and rules generated with Id 3 algorith $m$ are given thus:

\subsection{Id3 Tree}

TYREBURST $=$ TRUE

| $\mathrm{SEASON}=\mathrm{WET}$

| | TYPE = HAEVY VEHICLE

| | | TIME=EVENING: LOCATION2

| | | TIME = AFTERNOON: LOCATION2

| | | TIME=MORNING: LOCATION2

| | TIME = NIGHT: null

| | TYPE= SMALL CAR: LOCATION2

| TYPE = MOTOCYCLE: null

$\mathrm{SEASON}=\mathrm{DRY}$

| TIME $=$ EVENING

| | | TYPE= HAEVY VEHICLE: LOCATION2

| | | TYPE=SMALL CAR: LOCATION3

| | | TYPE = MOTOCYCLE: null

| $\mid$ TIME $=$ AFTERNOON

| | | TYPE = HAEVY VEHICLE: LOCATION2

| | | TYPE=SMALL CAR: LOCATION2

|| TYPE $=$ MOTOCYCLE: null

| $\mid$ TIME $=$ MORNING

| | | TYPE = HAEVY VEHICLE: LOCATION3

| | | TYPE=SMALL CAR: LOCATION3

| | | TYPE = MOTOCYCLE: null

TIME $=$ NIGHT: null

TYREBURST $=$ FALSE

TIME $=$ EVENING

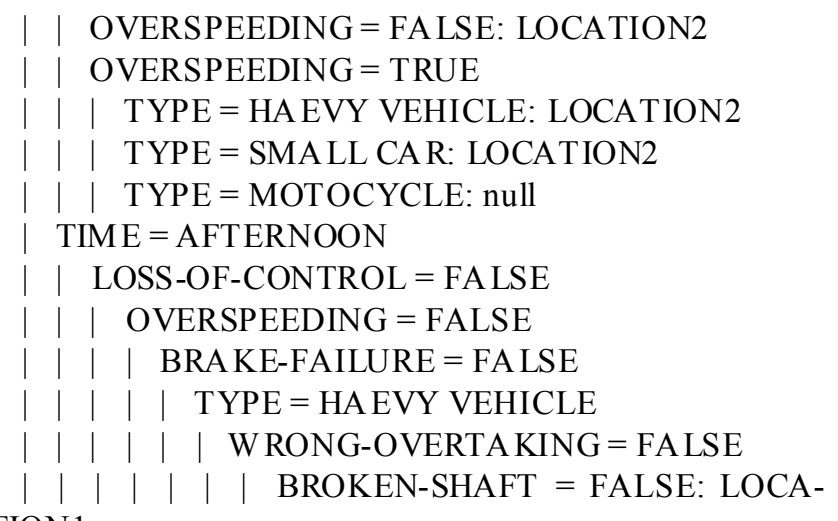

TION 1

| | | | | | | BROKEN-SHAFT = TRUE: LOCA-

TION3

l $|+| \mid$ WRONG-OVERTAKING = TRUE:

LOCATION2

| | | | TYPE= SMALL CAR

| | | | | SEASON =WET: LOCATION3

|||| $\mid$ SEASON = DRY

|||||| $\mid$ CARELESSDRIVING = FALSE:

LOCATION3

| | | | | | | CARELESSDRIVING = TRUE: LOCATION2

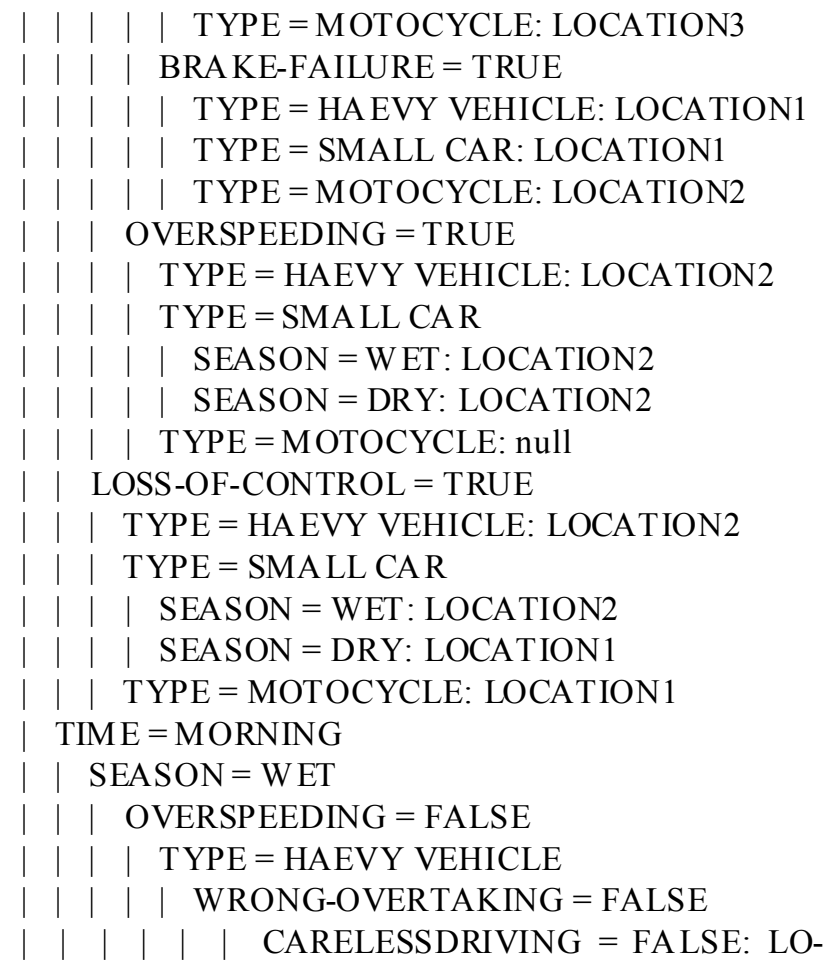

CATION1

| | | | | | CARELESSDRIVING = TRUE: LOCA-

TION2

| | | | | WRONG-OVERTAKING = TRUE: LOCATION 1

$\begin{array}{llllll}\mid & \mid & \mid & \text { TYPE }=\text { SMA LL CAR } \\ \mid & \mid & \mid & \mid & \text { CARELESSDRIVING = FALSE } \\ \mid & \mid & \mid & \mid & \text { | LOSS-OF-CONTROL = FA LSE: } \text { LOCA- }\end{array}$

TION3

LOSS-OF-CONTROL = TRUE: LOCA- 
TION2

|||| $\mid$ CARELESSDRIVING = TRUE: LOCATION1

| | | | TYPE=MOTOCYCLE: LOCATION2

| | | OVERSPEEDING = TRUE: LOCATION2

| $\mid$ SEASON = DRY

| | | BROKEN-SHAFT = FALSE

| | | | TYPE= HAEVY VEHICLE

| | | | | CARELESSDRIVING = FALSE

| | | | | LOSS-OF-CONTROL=FALSE

| | | | | | BROKEN-SPRING = FALSE

|||||||| OVERSPEEDING = FALSE: LO-

CATION2

| | | | | | | | OVERSPEEDING = TRUE: LOCA-

TION2

| | | | | | | BROKEN-SPRING = TRUE: LOCA-

TION2

| | | | | | LOSS-OF-CONTROL = TRUE: LOCA-

TION2

| $\mid$ | | | CARELESSDRIVING = TRUE: LOCA-

TION3

| | | TYPE = SMALL CAR

| | | | | CARELESSDRIVING = FALSE

| | | | | | | OVERSPEEDING = FALSE

| | | | | | | | UNKNOWN-CAUSES = FALSE

| | | | | | | | | | ROBBERY-ATTACK=FALSE

l $|+|$ | | | | WRONG-OVERTAKING =

FALSE

| | | | | | | | | | LOSS-OF-CONTROL=FALSE

l $|+||||| \mid$ | TREE-OBSTRUCTION =

FALSE

| | | | | | | | | | | | BRAKE-FAILURE =

FALSE: LOCATION3

| | | | | | | | | | | | BRAKE-FAILURE =

TRUE: LOCATION2

| | | | | | | | | | | | | TREE-OBSTRUCTION =

TRUE: LOCATION2

| | | | | | | | | | LOSS-OF-CONTROL=TRUE:

LOCATION2

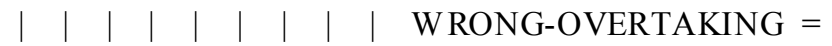

TRUE: LOCATION2

|||||||| ROBBERY-ATTACK = TRUE:

LOCATION3

| |||||| UNKNOWN-CAUSES = TRUE: LO-

CATION3

| | | | | | | | OVERSPEEDING = TRUE: LOCA-

TION3

| | | | | CARELESSDRIVING = TRUE: LOCA-

TION1

| | | | TYPE=MOTOCYCLE: null

| | BROKEN-SHAFT = TRUE: LOCATION3

TIME = NIGHT: LOCATION2

\section{Prism rules}

Rule 1 If BROKEN-SHAFT $=$ TRUE then LOCATION3

Rule 2 If ROBBERY-ATTACK $=$ TRUE and TYPE $=$ SMALL CA R then LOCATION3
Rule 3 If TREE-OBSTRUCTION $=$ TRUE and TIME $=$ EVENING then LOCATION3

Rule 4 If TYREBURST $=$ TRUE

and TIME $=$ MORNING

and TYPE $=$ SMALL CAR

and SEA SON $=$ DRY

and W RONG-OVERTA KING = FA LSE

and CARELESSDRIVING $=$ FALSE

and LOSS-OF-CONTROL $=$ FALSE

and OVERSPEEDING $=$ FALSE

and TREE-OBSTRUCTION $=$ FALSE

and PUSHED-BY-A-CAR $=$ FALSE

and BROKEN-SHAFT $=$ FALSE

and BROKEN-SPRING $=$ FALSE

and BRA KE-FAILURE $=$ FALSE

and ROAD-PROBLEM $=$ FALSE

and UNKNOWN-CA USES $=$ FALSE

and ROBBERY-ATTACK $=$ FALSE then LOCA-

TION3

Rule 5 If TYPE $=$ MOTOCYCLE

and CARELESSDRIVING $=$ TRUE then LOCA-

TION3

Rule 6 If ROAD-PROBLEM $=$ TRUE

and TYPE $=$ SMALL CAR

and TIME $=$ A FTERNOON

and SEASON $=$ DRY

and WRONG-OVERTAKING $=$ FALSE

and CARELESSDRIVING $=$ FALSE

and LOSS-OF-CONTROL $=$ FALSE

and TYREBURST $=$ FALSE

and OVERSPEEDING $=$ FALSE

and TREE-OBSTRUCTION $=$ FALSE

and PUSHED-BY-A-CAR $=$ FALSE

and BROKEN-SHAFT $=$ FALSE

and BROKEN-SPRING $=$ FALSE

and BRAKE-FAILURE $=$ FALSE

and UNKNOWN-CAUSES $=$ FALSE

and ROBBERY-ATTACK $=$ FALSE then LOCA-

TION3

Rule 7 If TYREBURST $=$ TRUE

and SEASON $=$ DRY

and TIME $=$ MORNING

and TYPE $=$ HAEVY VEHICLE

and WRONG-OVERTAKING $=$ FALSE

and CARELESSDRIVING $=$ FALSE

and LOSS-OF-CONTROL $=$ FALSE

and OVERSPEEDING $=$ FALSE

and TREE-OBSTRUCTION $=$ FALSE

and PUSHED-BY-A-CAR = FA LSE

and BROKEN-SHAFT $=$ FALSE

and BROKEN-SPRING $=$ FALSE

and BRA KE-FAILURE $=$ FALSE

and ROAD-PROBLEM $=$ FALSE

and UNKNOWN-CAUSES $=$ FALSE

and ROBBERY-ATTACK $=$ FALSE then LOCA-

TION3

Rule 8 If UNKNOWN-CAUSES $=$ TRUE 


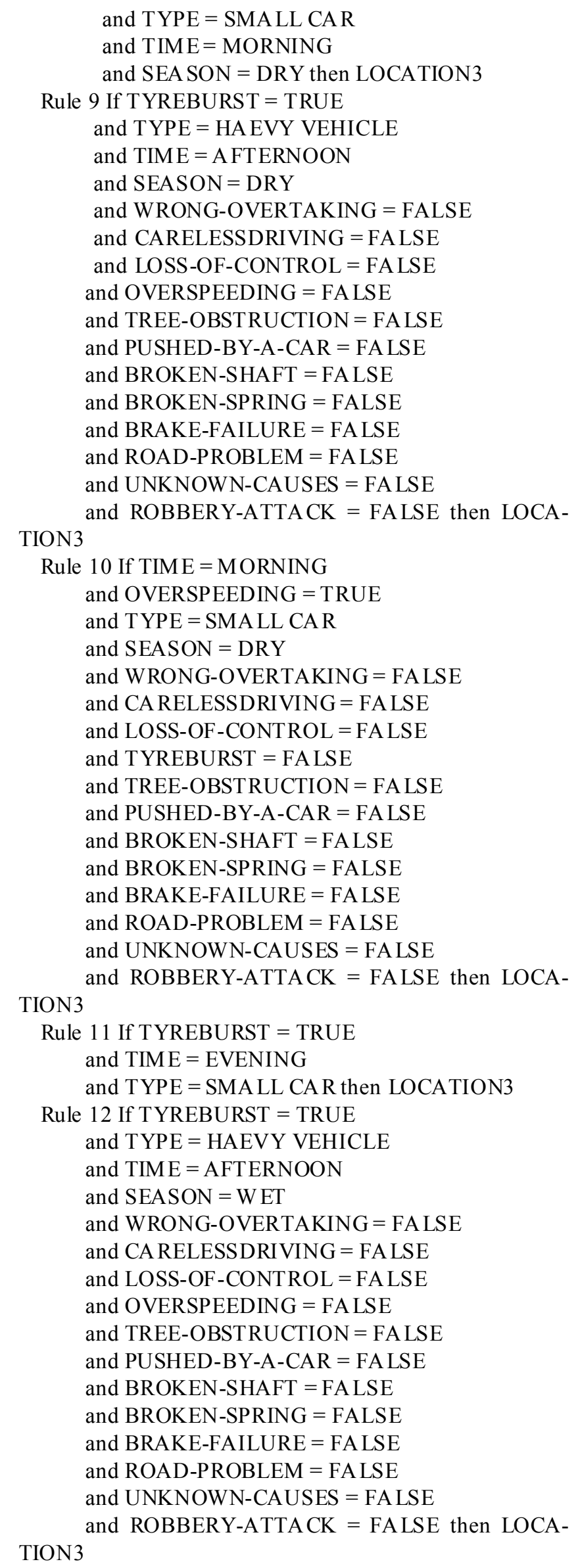

\section{Rule 13 If TIME $=$ MORNING}

and LOSS-OF-CONTROL $=$ TRUE

and TYPE $=$ HAEVY VEHICLE

and SEASON $=$ DRY

and WRONG-OVERTAKING $=$ FALSE

and CARELESSDRIVING $=$ FA LSE

and TYREBURST $=$ FALSE

and OVERSPEEDING $=$ FALSE

and TREE-OBSTRUCTION $=$ FALSE

and PUSHED-BY-A-CAR = FALSE

and BROKEN-SHAFT $=$ FALSE

and BROKEN-SPRING $=$ FALSE

and BRAKE-FAILURE $=$ FALSE

and ROAD-PROBLEM $=$ FALSE

and UNKNOWN-CAUSES $=$ FA LSE

and ROBBERY-ATTACK = FALSE then LOCA-

TION3

Rule 14 If UNKNOWN-CAUSES = TRUE

and TYPE $=$ SMALL CAR

and TIME $=$ MORNING

and SEASON $=$ WET

and WRONG-OVERTAKING $=$ FALSE

and CARELESSDRIVING $=$ FALSE

and LOSS-OF-CONTROL $=$ FALSE

and TYREBURST $=$ FA LSE

and TREE-OBSTRUCTION $=$ FALSE

and PUSHED-BY-A-CAR = FALSE

and BROKEN-SHAFT $=$ FALSE

and BROKEN-SPRING $=$ FALSE

and BRAKE-FAILURE $=$ FALSE

and ROAD-PROBLEM $=$ FALSE

and ROBBERY-ATTACK = FALSE then LOCA-

TION3

Rule 15 If TYREBURST $=$ TRUE

and TYPE $=$ HAEVY VEHICLE

and SEASON $=$ WET

and TIME $=$ EVENING

and WRONG-OVERTAKING $=$ FALSE

and CARELESSDRIVING $=$ FALSE

and OVERSPEEDING $=$ FALSE

and TREE-OBSTRUCTION $=$ FALSE

and PUSHED-BY-A-CAR $=$ FALSE

and BROKEN-SHAFT $=$ FALSE

and BROKEN-SPRING $=$ FALSE

and BRAKE-FAILURE $=$ FALSE

and ROAD-PROBLEM $=$ FALSE

and UNKNOWN-CAUSES $=$ FALSE

and ROBBERY-ATTACK $=$ FALSE then LOCA-

TION3

Rule 16 If TIME $=$ MORNING

and TYREBURST $=$ TRUE

and TYPE $=$ HAEVY VEHICLE

and SEASON $=$ W ET

and WRONG-OVERTAKING $=$ FALSE

and CARELESSDRIVING $=$ FALSE

and LOSS-OF-CONTROL $=$ FA LSE

and OVERSPEEDING $=$ FALSE 
and TREE-OBSTRUCTION $=$ FALSE

and PUSHED-BY-A-CAR $=$ FA LSE

and BROKEN-SHAFT $=$ FALSE

and BROKEN-SPRING $=$ FALSE

and BRAKE-FAILURE $=$ FALSE

and ROAD-PROBLEM $=$ FALSE

and UNKNOWN-CAUSES $=$ FALSE

and ROBBERY-ATTACK $=$ FALSE then LOCA-

TION3

Rule 17 If CA RELESSDRIVING $=$ TRUE

and TYPE $=$ HAEVY VEHICLE

and SEASON $=$ DRY then LOCATION3

Rule 18 If TIME $=$ MORNING

and TYPE $=$ SMALL CAR

and SEASON $=$ DRY

and CA RELESSDRIVING $=$ FA LSE

and WRONG-OVERTAKING = FALSE

and LOSS-OF-CONTROL $=$ FALSE

and TREE-OBSTRUCTION $=$ FALSE

and BRAKE-FAILURE = FALSE then LOCA-

TION3

Rule 19 If TIME $=$ NIGHT then LOCATION2

Rule 20 If W RONG-OVERTAKING = TRUE and TYPE $=$ SMALL CA R then LOCATION2

Rule 21 If TIME = EVENING and CARELESSDRIVING $=$ TRUE then LOCA-

TION2

Rule 22 If TIME $=$ EVENING

and UNKNOWN-CAUSES $=$ TRUE then LOCA-

TION2

Rule 23 If TIME $=$ EVENING

and LOSS-OF-CONTROL $=$ TRUE then LOCATION2

Rule 24 If TIME = EVENING

and ROBBERY-ATTACK = TRUE then LOCATION2

Rule 25 If TIME $=$ EVENING

and TYPE $=$ HAEVY VEHICLE

and SEASON $=$ DRY then LOCATION2

Rule 26 If SEASON $=$ WET

and TYPE $=$ MOTOCYCLE then LOCATION2

Rule 27 If SEASON $=$ WET

and OVERSPEEDING $=$ TRUE

and TIME $=$ MORNING then LOCATION2

Rule 28 If TYREBURST $=$ TRUE

and SEASON $=$ W ET

and TYPE $=$ SMALL CA R then LOCATION2

Rule 29 If TYREBURST $=$ TRUE

and SEASON $=\mathrm{WET}$

and TIME $=$ MORNING

and TYPE $=$ HAEVY VEHICLE

and WRONG-OVERTAKING $=$ FALSE

and CARELESSDRIVING $=$ FALSE

and LOSS-OF-CONTROL $=$ FALSE

and OVERSPEEDING $=$ FALSE

and TREE-OBSTRUCTION = FALSE

and PUSHED-BY-A-CAR $=$ FALSE and BROKEN-SHAFT $=$ FALSE

and BROKEN-SPRING $=$ FALSE

and BRAKE-FAILURE $=$ FALSE

and ROAD-PROBLEM = FALSE

and UNKNOWN-CAUSES $=$ FA LSE

TION2

and ROBBERY-ATTACK = FALSE then LOCA-

Rule 30 If TYPE $=$ HAEVY VEHICLE

and ROBBERY-ATTACK = TRUE then LOCA-

TION2

Rule 31 If TYPE $=$ HAEVY VEHICLE and OVERSPEEDING $=$ TRUE

and TIME $=$ AFTERNOON then LOCATION2

Rule 32 If TYREBURST $=$ TRUE

and SEASON $=$ WET

and TIME $=$ EVENING

and TYPE $=$ HAEVY VEHICLE

and WRONG-OVERTAKING $=$ FA LSE

and CARELESSDRIVING $=$ FA LSE

and LOSS-OF-CONTROL $=$ FALSE

and OVERSPEEDING $=$ FALSE

and TREE-OBSTRUCTION $=$ FALSE

and PUSHED-BY-A-CAR = FALSE

and BROKEN-SHAFT $=$ FALSE

and BROKEN-SPRING $=$ FALSE

and BRAKE-FAILURE $=$ FA LSE

and ROAD-PROBLEM $=$ FALSE

and UNKNOWN-CAUSES $=$ FA LSE

and ROBBERY-ATTACK $=$ FALSE then LOCA-

TION2

Rule 33 If TYREBURST $=$ TRUE

and SEASON $=$ WET

and TYPE $=$ HAEVY VEHICLE

and TIME $=$ AFTERNOON

and WRONG-OVERTAKING $=$ FALSE

and CARELESSDRIVING $=$ FALSE

and LOSS-OF-CONTROL $=$ FALSE

and OVERSPEEDING $=$ FALSE

and TREE-OBSTRUCTION $=$ FALSE

and PUSHED-BY-A-CAR $=$ FALSE

and BROKEN-SHAFT $=$ FALSE

and BROKEN-SPRING $=$ FALSE

and BRAKE-FAILURE $=$ FALSE

and ROAD-PROBLEM $=$ FALSE

and UNKNOWN-CAUSES $=$ FALSE

and ROBBERY-ATTACK = FALSE then LOCA-

TION2

Rule 34 If TYPE = HAEVY VEHICLE

and TIME $=$ EVENING then LOCATION2

Rule 35 If TYPE $=$ HAEVY VEHICLE

and OVERSPEEDING $=$ TRUE

and TIME $=$ MORNING

and SEASON $=$ DRY

and WRONG-OVERTAKING $=$ FALSE

and CA RELESSDRIVING $=$ FA LSE

and LOSS-OF-CONTROL $=$ FALSE

and TYREBURST $=$ FALSE 
and TREE-OBSTRUCTION $=$ FALSE

and PUSHED-BY-A-CAR $=$ FA LSE

and BROKEN-SHAFT $=$ FALSE

and BROKEN-SPRING $=$ FALSE

and BRAKE-FAILURE $=$ FALSE

and ROAD-PROBLEM = FALSE

and UNKNOWN-CAUSES $=$ FALSE

and ROBBERY-ATTACK $=$ FALSE then LOCATION2

Rule 36 If TYREBURST $=$ TRUE

and TIME $=$ AFTERNOON

and TYPE $=$ SMALL CAR

and SEASON $=$ DRY

and WRONG-OVERTAKING $=$ FALSE

and CARELESSDRIVING $=$ FALSE

and LOSS-OF-CONTROL $=$ FALSE

and OVERSPEEDING $=$ FALSE

and TREE-OBSTRUCTION $=$ FALSE

and PUSHED-BY-A-CAR = FALSE

and BROKEN-SHAFT $=$ FALSE

and BROKEN-SPRING $=$ FALSE

and BRAKE-FAILURE $=$ FALSE

and ROAD-PROBLEM $=$ FALSE

and UNKNOWN-CAUSES $=$ FALSE

and ROBBERY-ATTACK = FALSE then LOCA-

TION2

Rule 37 If BRAKE-FA ILURE $=$ TRUE and TYPE $=$ MOTOCYCLE then LOCATION2

Rule 38 If W RONG-OVERTAKING = TRUE and TIME $=$ AFTERNOON then LOCATION2

Rule 39 If TREE-OBSTRUCTION $=$ TRUE and TIME $=$ MORNING then LOCATION2

Rule 40 If BROKEN-SPRING $=$ TRUE and TYPE $=$ HAEVY VEHICLE

and TIME $=$ MORNING

and SEASON $=$ DRY

and WRONG-OVERTAKING $=$ FALSE

and CA RELESSDRIVING $=$ FALSE

and LOSS-OF-CONTROL $=$ FALSE

and TYREBURST $=$ FALSE

and OVERSPEEDING $=$ FALSE

and TREE-OBSTRUCTION $=$ FALSE

and PUSHED-BY-A-CAR $=$ FA LSE

and BROKEN-SHAFT $=$ FALSE

and BRAKE-FAILURE $=$ FALSE

and ROAD-PROBLEM $=$ FALSE

and UNKNOWN-CAUSES $=$ FALSE

and ROBBERY-ATTACK = FALSE then LOCA-

TION2

Rule 41 If TYPE = HAEVY VEHICLE

and TYREBURST $=$ TRUE

and TIME $=$ AFTERNOON

and SEASON $=$ DRY

and WRONG-OVERTAKING $=$ FALSE

and CA RELESSDRIVING $=$ FALSE

and LOSS-OF-CONTROL $=$ FA LSE

and OVERSPEEDING $=$ FALSE and TREE-OBSTRUCTION $=$ FALSE

and PUSHED-BY-A-CAR = FALSE

and BROKEN-SHAFT $=$ FALSE

and BROKEN-SPRING $=$ FALSE

and BRAKE-FAILURE $=$ FALSE

and ROAD-PROBLEM $=$ FA LSE

and UNKNOWN-CAUSES $=$ FALSE

and ROBBERY-ATTACK $=$ FALSE then LOCA-

TION2

Rule 42 If LOSS-OF-CONTROL $=$ TRUE

and TIME $=$ MORNING

and TYPE $=$ SMALL CAR then LOCATION2

Rule 43 If UNKNOWN-CAUSES $=$ TRUE

and TYPE $=$ HAEVY VEHICLE

and SEASON $=$ DRY then LOCATION2

Rule 44 If OVERSPEEDING $=$ TRUE

and TIME $=$ AFTERNOON

and SEASON $=$ WET then LOCATION2

Rule 45 If TYPE $=$ HAEVY VEHICLE

and LOSS-OF-CONTROL $=$ TRUE

and TIME $=$ MORNING

and SEASON $=$ DRY

and WRONG-OVERTAKING $=$ FALSE

and CARELESSDRIVING $=$ FALSE

and TYREBURST $=$ FALSE

and OVERSPEEDING $=$ FALSE

and TREE-OBSTRUCTION $=$ FALSE

and PUSHED-BY-A-CAR = FALSE

and BROKEN-SHAFT $=$ FALSE

and BROKEN-SPRING $=$ FALSE

and BRAKE-FAILURE $=$ FALSE

and ROAD-PROBLEM $=$ FALSE

and UNKNOWN-CAUSES $=$ FA LSE

and ROBBERY-ATTACK = FALSE then LOCA-

TION2

Rule 46 If SEASON = WET

and LOSS-OF-CONTROL $=$ TRUE

and TIME $=$ AFTERNOON

and WRONG-OVERTAKING $=$ FALSE

and CARELESSDRIVING $=$ FALSE

and TYREBURST $=$ FALSE

and OVERSPEEDING $=$ FALSE

and TREE-OBSTRUCTION $=$ FALSE

and PUSHED-BY-A-CAR = FALSE

and BROKEN-SHAFT $=$ FALSE

and BROKEN-SPRING $=$ FALSE

and BRAKE-FAILURE $=$ FALSE

and ROAD-PROBLEM $=$ FALSE

and UNKNOWN-CAUSES $=$ FALSE

and ROBBERY-ATTACK $=$ FALSE

and TYPE $=$ HAEVY VEHICLE then LOCATION2

Rule 47 If CA RELESSDRIVING $=$ TRUE

and TIME $=$ AFTERNOON

and TYPE $=$ SMALL CA R then LOCATION2

Rule 48 If OVERSPEEDING $=$ TRUE

and TIME $=$ AFTERNOON

and TYPE $=$ SMALL CAR 
and SEASON $=$ DRY

and WRONG-OVERTAKING $=$ FALSE

and CARELESSDRIVING $=$ FALSE

and LOSS-OF-CONTROL $=$ FALSE

and TYREBURST $=$ FALSE

and TREE-OBSTRUCTION $=$ FALSE

and PUSHED-BY-A-CAR $=$ FALSE

and BROKEN-SHAFT $=$ FALSE

and BROKEN-SPRING $=$ FALSE

and BRAKE-FAILURE $=$ FALSE

and ROAD-PROBLEM $=$ FALSE

and UNKNOWN-CAUSES $=$ FALSE

and ROBBERY-ATTACK $=$ FALSE then LOCA-

TION2

Rule 49 If SEASON $=$ WET

and TIME $=$ EVENING

and TYPE $=$ SMA LL CA R

and WRONG-OVERTAKING $=$ FALSE

and CARELESSDRIVING $=$ FALSE

and LOSS-OF-CONTROL $=$ FALSE

and TYREBURST $=$ FALSE

and OVERSPEEDING $=$ TRUE

and TREE-OBSTRUCTION $=$ FALSE

and PUSHED-BY-A-CAR = FALSE

and BROKEN-SHAFT $=$ FALSE

and BROKEN-SPRING $=$ FALSE

and BRAKE-FAILURE $=$ FALSE

and ROAD-PROBLEM $=$ FALSE

and UNKNOWN-CAUSES $=$ FALSE

TION2

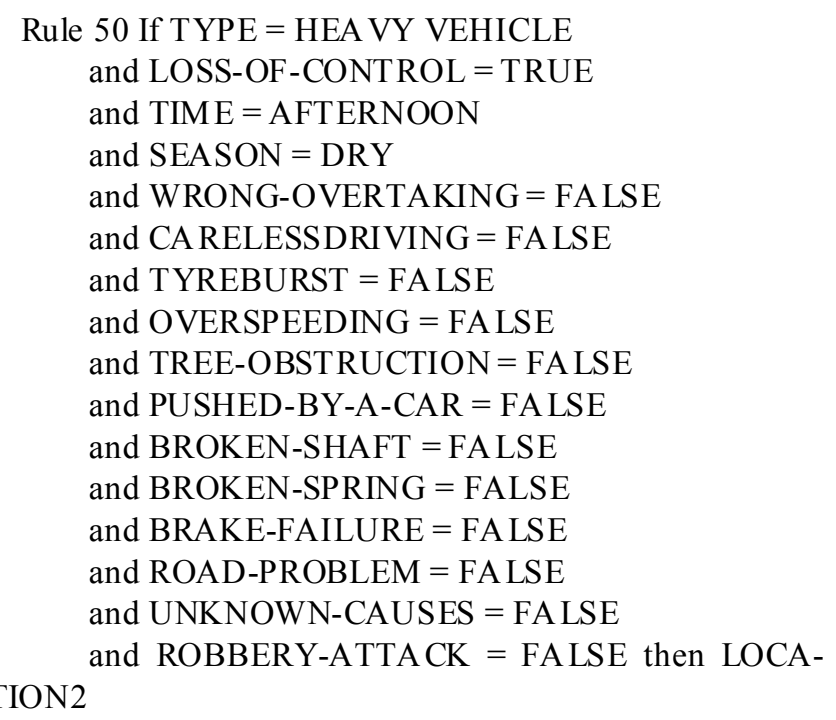

\section{Discussion}

There are 50 rules generated from this tree. Rule 1- 18 indicate the occurrence of accident in Location 3 and rule 19-50 also shows the occurrence of accident in location 2.This indicate that, location 2 has the highest number of road accident occurrence with Heavy-vehicle in the afternoon and during the dry season.

Rule 41 is the best one that can be used for prediction. The rule says that, Tyre bust is the cause of road accident with heavy vehicle within location 2 in the day time and during the dry season.

\section{Decision Tree Performance Analysis on Id3}

Table 5.1. Detailed Accuracy By class

\begin{tabular}{|c|c|c|c|c|c|c|}
\hline Class & TP rate & FT rate & Precision & Recall & F- measure & Roc Area \\
\hline Location (3) & 0.688 & 0.069 & 0.733 & 0.688 & 0.71 & 0.942 \\
\hline Location (2) & 0.897 & 0.361 & 0.78 & 0.897 & 0.834 & 0.888 \\
\hline Location (1) & 0.517 & 0.025 & 0.833 & 0.517 & 0.638 & 0.95 \\
\hline Weighted Avg. & 0.777 & 0.232 & 0.78 & 0.777 & 0.769 & 0.912 \\
\hline
\end{tabular}

Table 5.2. Confusion matrix Predicted category

\begin{tabular}{|c|c|c|c|}
\hline Actual category & Location (3) & Location (2) & Location (1) \\
\hline Location (3) & 22 & 10 & 0 \\
\hline Location (2) & 6 & 78 & 3 \\
\hline Location (1) & 2 & 12 & 15 \\
\hline
\end{tabular}

Decision Tree perform ance Analysis on Function Tree (FT)

Table 5.3. Detailed Accuracy by Class

\begin{tabular}{|c|c|c|c|c|c|c|}
\hline Class & TP rate & FT rate & Precision & Recall & F- measure & Roc Area \\
\hline Location (3) & 0.625 & 0.086 & 0.667 & 0.625 & 0.645 & 0.869 \\
\hline Location (2) & 0.77 & 0.361 & 0.753 & 0.77 & 0.761 & 0.736 \\
\hline Location (1) & 0.586 & 0.101 & 0.586 & 0.586 & 0.586 & 0.832 \\
\hline Weighted Avg. & 0.703 & 0.25 & 0.702 & 0.703 & 0.702 & 0.783 \\
\hline
\end{tabular}

Table 5.4. Confusion Matrix Predicted category

\begin{tabular}{|c|c|c|c|}
\hline Actual category & Location (3) & Location (2) & Location (1) \\
\hline Location (3) & 20 & 12 & 0 \\
\hline Location (2) & 8 & 67 & 12 \\
\hline Location (1) & 2 & 10 & 17 \\
\hline
\end{tabular}




\section{Conclusions}

Using WEKA software to analy ze accident data collected on Lagos-Ibadan road, it was found that decision tree can accurately predict the cause(s) of accident and accident prone locations along the road and other roads if relevant data are gathered and analyzed as in this case.

In Decision Tree Performance analysis, the, datas et were experimented with two algorith ms; Id 3 and FT (function tree) For Id 3 algorithm, there were 115 correctly classified instances and 33 incorrectly classified instances which represent $77.70 \%$ and $22.29 \%$ respectively. Mean absolute error was 0.1835 and Root mean squared error was 0.3029 .

Also for functional tree algorithm (FT), total number of tree size was 5 with 105 correctly classified instances representing $70.27 \%$ and 44 incorrectly classified instances representing $29.73 \%$.

From the detailed accuracy by class and confusion matrix, Id 3 attained accuracy rate of 0.777 and FT attained accuracy rate of 0.703 .

\section{REFERENCES}

[1] Akomolafe et al (2009) "Enhancing road monitoring and safety through the use of geo spatial technology" International Journal of Physical Sciences Vol. 4 (5), pp. 343-348
[2] Akomolafe, O.P. (2004); predicting possibilities of Road Accidents occurring, using Neural Network. M. Sc. Thesis, Department of Computer Science, University of Ibadan

[3] Abdalla, I.M., Robert, R., Derek, B. and McGuicagan, D.R.D.,(1987) An investigation into the relationships between area social characteristics and road accident casualties. Accid. Anal prev. 29 5, pp. 583-593, 1997

[4] Gelfand, S.G., Ravishanker, C.S., and Delp, E.J.(1991) An iterative Growing and Pruning Algorithm for Classification Tree Design, PAMI(13), No. 2, February 1991, pp. 163-174

[5] Han J. and Kamber M. (2001) Data mining Concepts and Techniques Morgan Kaufmam, Academic Press

[6] Han J. and Kamber M. (2001) Data mining Concepts and Techniques Morgan Kaufmam, Academic Press

[7] Hand, D., Mannila, H., \& Smyth, P., (2001) Princip les of data Mining. The MIT Press, 2001

[8] Kim, K., Nitz, L., Richardson, J., \& Li, L., (1995) Personal and Behavioral Predictors of Automobile Crash and Injury Severity. Accident Analysis and Prevention, Vol. 27, No. 4, 1995, pp. 469-481

[9] Martin, P. G., Crandall, J. R., \& Pilkey, W. D.,(2000) Injury Trends of Passenger Car Drivers in the USA Accident Analysis and Prevention, Vol. 32, 2000, pp. 541-557

[10] Ossenbruggen, P.J., pendharkar, J. and Ivan, J., (2001) Roadway safety in rural and small urbanized areas. Accid. Anal. Prev. 334, pp. 485-498, 2001 